\title{
Visceral leishmaniasis in an immunocompetent patient
}

\author{
Domingos Sousa $^{1}$, Miguel Seruca ${ }^{1}$, Ana Isabel Rodrigues ${ }^{1}$, Mourão Carvalho ${ }^{1}$, Sara Aleixo \\ Duarte $^{1}$, Sérgio Antunes Silva ${ }^{1}$, Micael Mendes ${ }^{1}$, Alejandra Pereira ${ }^{1}$, Amparo Mingo ${ }^{1}$, \\ Elena Rios ${ }^{1}$, and Mário Lázaro ${ }^{1}$ \\ ${ }^{1}$ Centro Hospitalar do Algarve EPE
}

December 21, 2020

\begin{abstract}
A 26-year-old patient was admitted with a 6-month history of fever,fatigue,and unintentional weight loss.Abdominal CT described an heterogeneous hepatosplenomegaly.Laboratory studies revealed leucopenia,anemia and elevated CRP.Bone marrow aspirate revealed amastigotes compatible with Leishmania spp. Was treated with liposomal amphotericin B with favourable outcome.Authors intend to raise awareness of VL in immunocompetentpatients.
\end{abstract}

Title: Visceral leishmaniasis in an immunocompetent patient

Domingos Sousa $^{1}$, Miguel Seruca ${ }^{2}$, Ana Isabel Rodrigues ${ }^{1}$, Mourão Carvalho ${ }^{1}$, Sara Aleixo Duarte ${ }^{1}$, Sérgio Antunes Silva ${ }^{1}$, Micael Mendes ${ }^{1}$, Alejandra Pereira ${ }^{2}$, Amparo Mingo $^{3}$, Elena Rios ${ }^{1}$, Mário Lázaro ${ }^{1}$

${ }^{1}$ Internal Medicine Department, Centro Hospitalar e Universitário do Algarve, Unidade de Faro, Portugal

${ }^{2}$ Clinical Pathology Department, Centro Hospitalar e Universitário do Algarve, Unidade de Faro, Portugal.

${ }^{3}$ Infeccious Diseases Department, Centro Hospitalar e Universitário do Algarve, Unidade de Faro, Portugal.

Email address of Corresponding author: domingos.sousaa@gmail.com

orcid.org/ 0000-0001-6657-5921 telephone: +351 913334854

Key clinical words: fever, anemia, leishmaniasis, immunocompetent

Key clinical message: Visceral leishmaniasis in immunocompetent patients is rare and should be suspected in patients with fever, bicytopenia and elevated inflammatory markers.

A previously healthy man with 26-year-old presented to the emergency department with a 6-month history of fever, fatigue and an unintentional 12 kilogram weight loss. At physical examination, an enlarged spleen was notable. Further studies revealed a leucocyte count of 2200 per cubic millimeter, a hemoglobin level of $9.4 \mathrm{~g}$ per deciliter, a platelet count of 200,000 per cubic millimeter and hyperglobulinemia. Blood cultures as well as serologies for human immunodeficiency virus, hepatitis B virus, hepatitis C virus, Epstein-Barr virus, and cytomegalovirus came back negative. Computed tomography (CT) of the abdomen confirmed the presence of a heterogeneous hepatosplenomegaly (Figure 1A). Examination of a bone marrow aspirate revealed the presence of amastigotes within the macrophages' cytoplasm, a classic appearance of Leishmania spp ( Figure 1B). Leishmaniasis is a globally widespread zoonosis that is transmitted by the bite of an infected female phlebotomine sandfly. Domestic dogs are the parasite reservoirs. Clinically, leishmaniasis is subdivided into cutaneous, mucocutaneous and visceral (kala-azar) forms. The most severe form is Visceral leishmaniasis(VL) that is characterized by a a disseminated intracellular protozoan infection that targets tissue macrophages in the liver, spleen and bone marrow. BothLeishmania infantum and Leishmania donovani can cause VL, 
being Leishmania infantum the most prevalent pathogen in Portugal and remaining Mediterranean countries. The patient started treatment with liposomal amphotericin B leading to complete symptom resolution and splenomegaly on physical examination. Moreover, the differential diagnosis in a patient with fever and splenomegaly must include other infective causes such as typhoid, tuberculosis, leptospirosis; autoimmune disorders such as rheumatoid arthritis, sarcoidosis, amyloidosis and systemic erythematous lupus and haematological disorders such as leukaemia, lymphoma, polycythaemia vera and myelofibrosis. The present report intends to raise awareness of VL in immunocompetent patients that should be included in the diagnostic workup of patients with splenomegaly.

\section{Compliance with ethical standards}

Conflicts of interest: None to declare.

Human and animal rights: This article does not contain any study with human and animals performed by any of the authors.

Informed consent: Informed consent was signed.

Contributors DS and MS: acquisition of data, literature review, clinical and imaging data review and final manuscript writing. AIR, MC, SAD, SAS, MM, AP, AM, ER and ML: imaging data review, and final manuscript writing.

\section{Funding}

Authors do not have any funding to declare for the present case report.

\section{Acknowledgement statement}

The authors want to acknowledge to all the members of the Radiology and Clinical Pathology department for the support in the appropriate imaging collection.

\section{References:}

1- Campino, L. and Maia, C. (2010) 'Epidemiologia das leishmanioses em Portugal', Acta Medica Portuguesa , 23(5), pp. 859-864.

2- van Griensven, J. and Diro, E. (2019) 'Visceral Leishmaniasis: Recent Advances in Diagnostics and Treatment Regimens',Infectious Disease Clinics of North America . Elsevier Inc, 33(1), pp. 79-99. doi: 10.1016/j.idc.2018.10.005.

\section{Figures Legends:}

1A: CT sagittal images shows and heterogenous hepatosplenomegaly with a $18 \mathrm{~cm}$ spleen.

1B: Leishmania spp. in May Grunwald Giemsa (MGG) stainded bone marrow aspirate smear. Multiple amastigotes are visible in the macrophage cytoplasm, exhibiting a nucleus (blue arrow) and a kinetoplast (red arrow). 


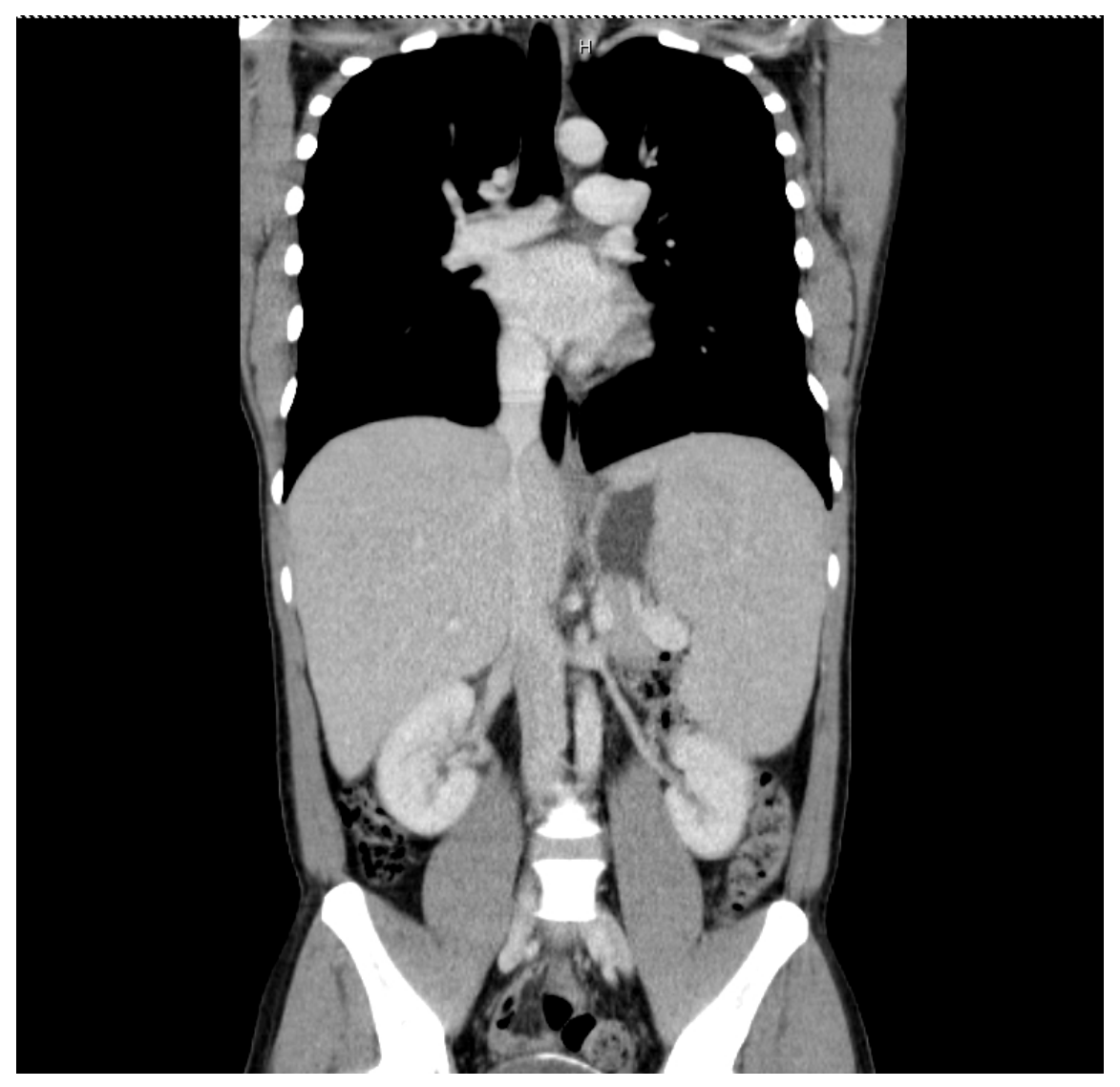




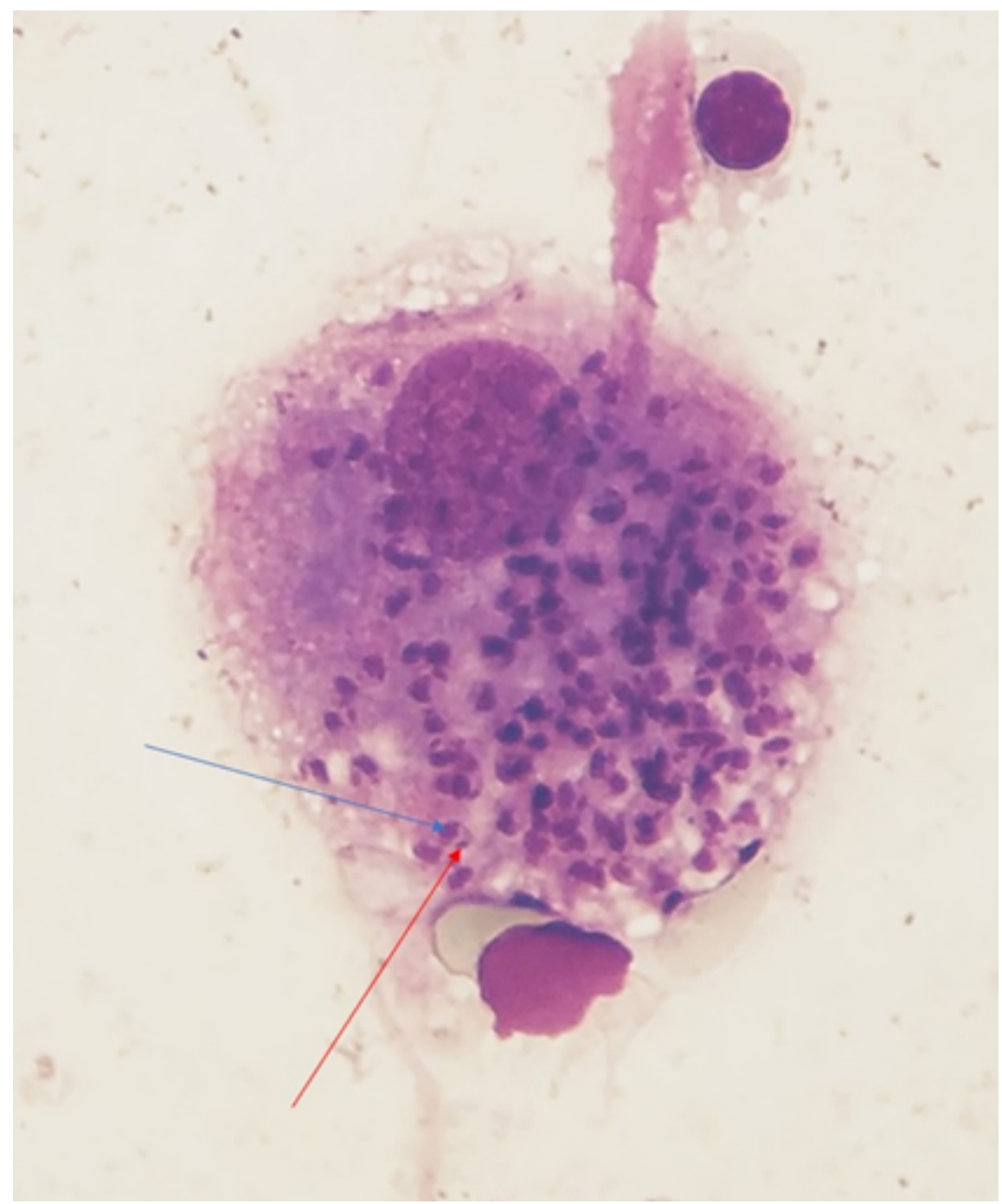

